\title{
Global Health Epidemic (Earthquake to Road Traffic Accident): Innovative Disaster Pedagogy Harnessing Technology
}

\author{
Dinesh Vyas ${ }^{1,2 *}$, Kaivalya Deshpande ${ }^{3}$ and Arpita Vyas ${ }^{2}$ \\ ${ }^{1}$ Surgery Residency, San Joaquin General Hospital, CA \\ ${ }^{2}$ California North State University \\ ${ }^{3}$ University of Pittsburgh
}

*Corresponding author: Dinesh Vyas, Department of Surgery, San Joaquin General Hospital, French Camp, CA

\begin{tabular}{|c|c|}
\hline ARTICLE INFO & ABSTRACT \\
\hline 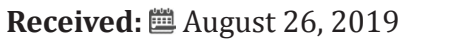 & Citation: Dinesh V, Kaivalya D, Arpita V. Global Health Epidemic (Earthquake to Road \\
\hline Published: 慧 September 03, 2019 & $\begin{array}{l}\text { Traffic Accident): Innovative Disaster Pedagogy Harnessing Technology. Biomed J Sci \& } \\
\text { Tech Res 21(1)-2019. BJSTR. MS.ID.003545. }\end{array}$ \\
\hline
\end{tabular}

\section{Editorial}

\section{Background}

One of the significant driving factors behind global trauma morbidity and mortality is underdeveloped and underrepresented pre-hospital care services in areas of need. These include remote areas in developed nations, urban area in fast growing economies or natural disaster in any part of world. Natural disasters are becoming more common with fires and earthquakes striking more frequently. Multi-center studies conducted by Dr. Dinesh Vyas have demonstrated that the root of the issue stems from the lack of a pragmatic, contextual, well-researched and accessible training paradigm, applicable to both developed and underdeveloped settings. Training this efficient workforce must address the constituent's need with a pedagogy immersed in technology including and not limited to animations and augmented reality.

The problem stems from the delay in response time after a traumatic incident, leading to the highest mortality rates occurring within the first $30-60 \mathrm{~min}$ of an accident-putting greater emphasis on the role of a first responder [1]. The absence of an effective training paradigm, as well as the increasing burden of costs and scarcity of knowledgeable personnel severely contributes to an ineffective system of trauma care. The novel education program (innovation in every component of education) and its implementation in nine international centers generated promising results in competency, confidence, psychosomatic skills and retention of knowledge. Our well-established program has approached this dilemma through the advent of novel training schemes, integrating technology (pretraining online education using engaging animation videos using professional expert in mass communication) and multitude of virtual interfaces with hands-on training targeting various nonmedical personnel [2,3]. With the rapidly growing technology sector providing ubiquitous access to developing settings across the globe, integrating this avenue in trauma training becomes paramount. Through the use of high-fidelity simulation, body camera recording and feedback, mock drill with video debriefing, virtual skill assessment, and a self-perpetuating training paradigm, Vyas et al have successfully remodeled the landscape of trauma training at various developing sites across the world such as India and the rural setting in the western world such as West Texas [4].

As shown in Figure 1, the global trauma-care initiative strives to maximize the post-trauma incident role of the layperson first responder, thus effectively reducing system and hospital burden and minimizing immediate motor vehicle accident complications. By providing training to existing non-medical workforce local policemen, volunteer firefighters, truck and Taxi drivers, local educators/teachers, safety managers and other appropriate roadside faculty, the onset of victim care begins immediately after the accident. A pre-implementation research of need analysis is done for development of education modules in the local language, identification of trauma pertinent local myths, accident-prone 
locations, and population density. Ultimately, the sustainable and replicable model of education can be integrated with current trauma- training regimens in hopes of enhancing trauma management capacity and standardized high-quality care in urbanized settings.

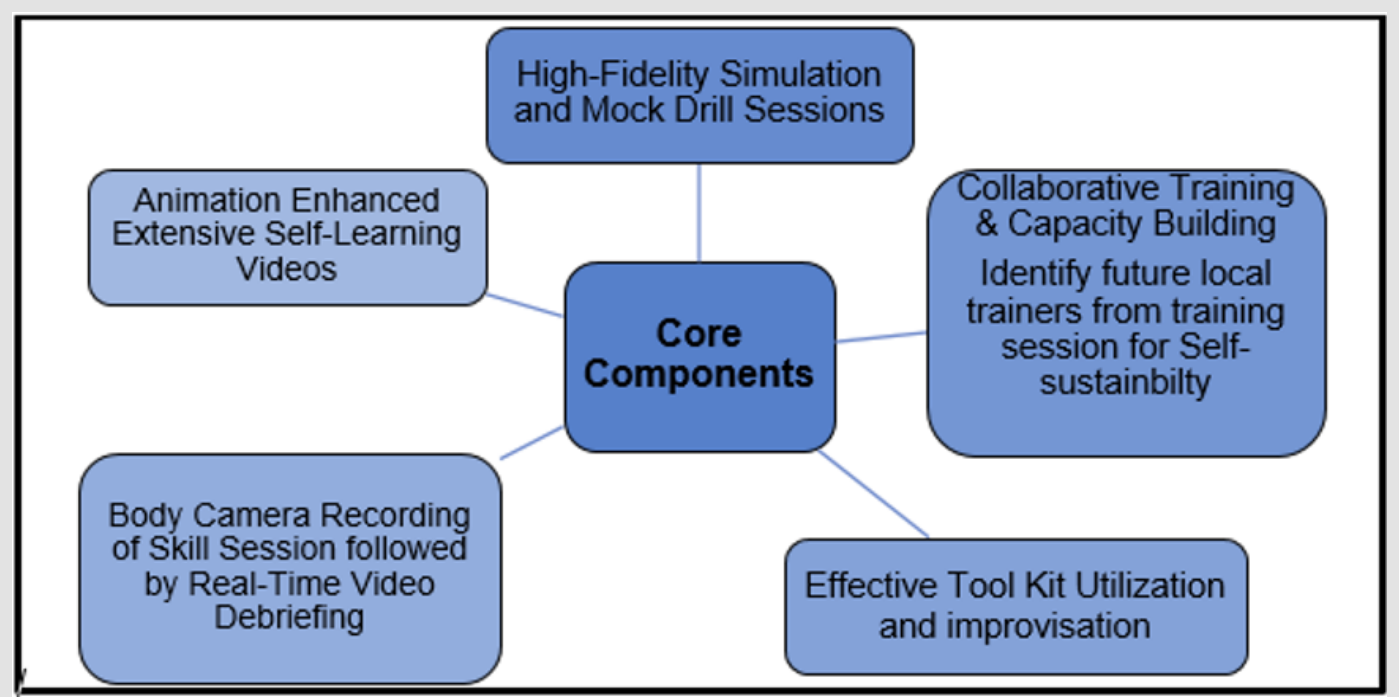

Figure 1: Core Components of the Global Trauma Initiative: Training of Trainer.

\section{Integrated Technology Using a 4-Tiered Protocol}

Building off currently established training designs [5], the initiative designed by Vyas et al. follows a standardized multiphase model individualized to different trauma settings. Starting with the Massively Open Online Course (MOOC), the first phase establishes the necessary pre-requisites for first-responder trainees. Using the gold-standard guidelines developed by training courses such as Basic Life Support, Acute Trauma Life Support, International Trauma Life Support, Acute Care Life Support and Pre-hospital Trauma Life Support [6], the MOOC phase eliminates the need for costly non-standardized human instruction, and effectively targets a broad range of professionals. MOOC has generated audience from more than 2000 first responders from 37 countries in 2015.

The subsequent tiers, Acute Trauma Training (ATT), Broad Trauma Training (BTT), and Cardiac Trauma Training (CTT), each involves the instruction of progressively advanced skills (Table 1) and utilizes an algorithm to determine proper trainee advancement [7]. Each tier involves various online as well as hands-on components. Using virtual simulators and instructional videos, the modules strive to mimic real world post-accident conditions. Skills such as airway management, stabilization, assessment of the scene, hemorrhage control, fracture, chest injury, burn, extrications, explosions etc. are introduced using the online interface and supplemented with hands on training sessions. This integration of technology not only addresses the scarcity of trained personnel and resources but also provides individualized pacing and self-assessment opportunities for the trainee. This allows for trainees of different backgrounds, such as policemen, firefighters, etc. to play a direct role in trauma management. Post-training competence assessment analysis demonstrated a statistically significant increase in first responder trauma-management capabilities (Figures 2-4) [8].

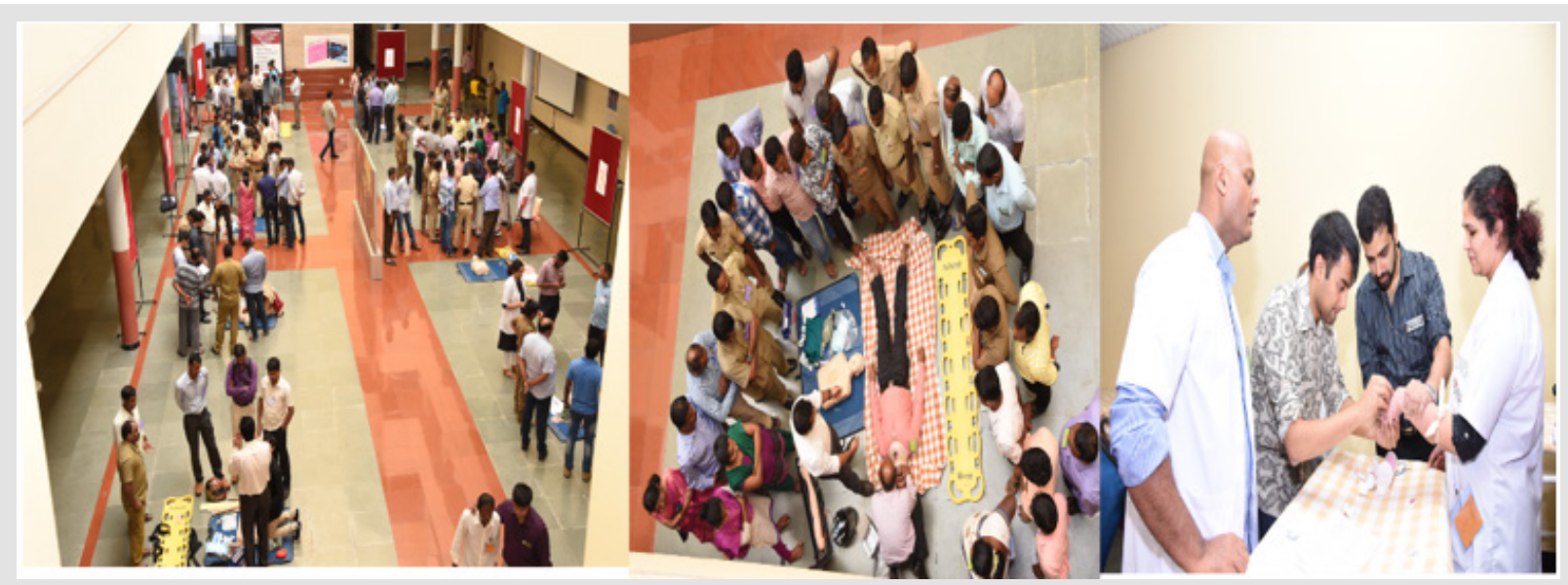

Figure 2: Establishing training program: Overview showing 10 training centers of skills (left), Bird's eye view of each group (middle); and skill learning with one to one feedback for each trainee (right). 


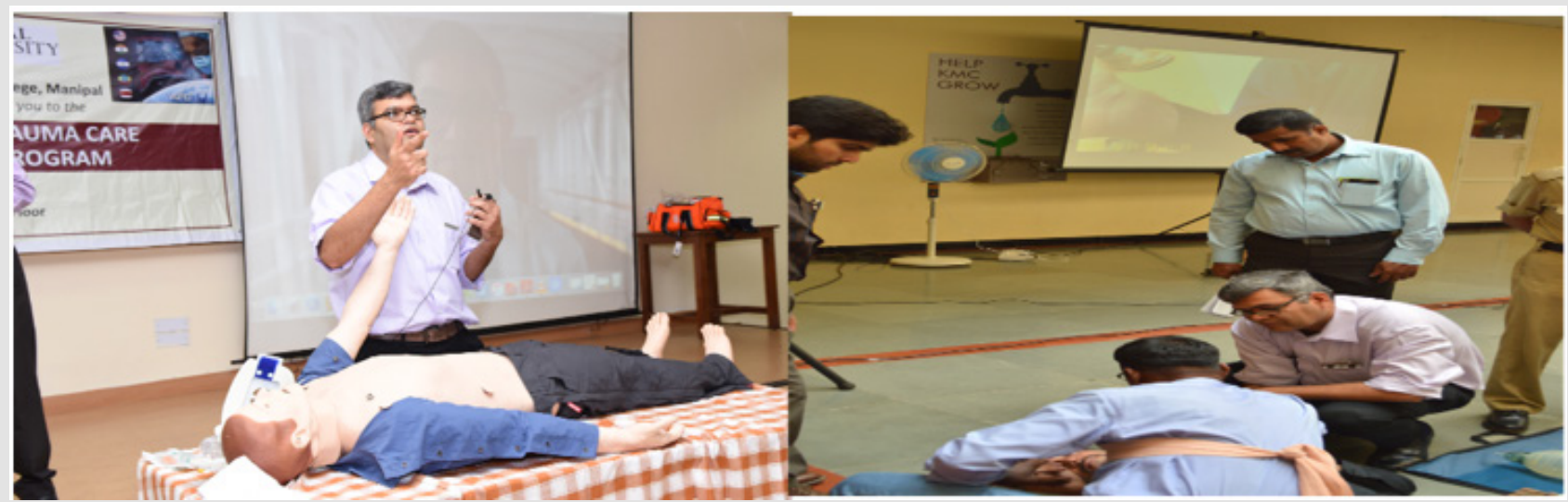

Figure 3: Technology integration: Use of high-fidelity simulators and other tool (left); Use of innovative low-cost body camera (wi-fi enabled) with ACE wrap harness for chest mount. Screen on the background displaying the skills and recording the session.

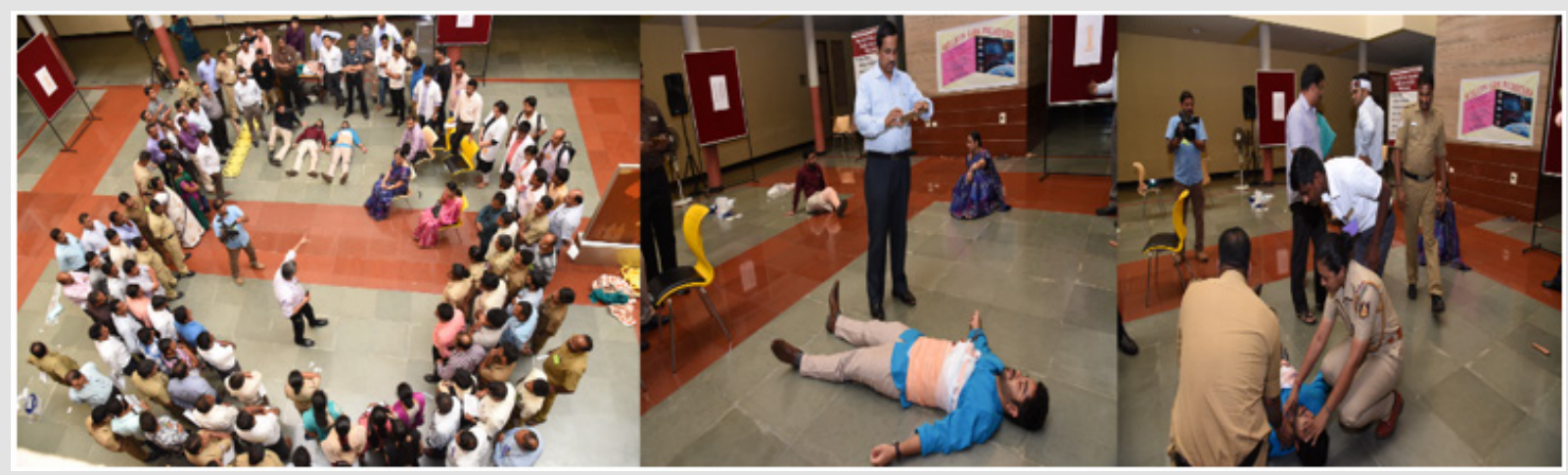

Figure 4: Mock drill for psychosomatic training: Simulating enviornmet of polytraumas and road side chaos (left); Initial stages of trauma and public response (middle); First responders using the skills after 10hr training (right).

Table 1: Context Specific Skills in each Program Tier.

\begin{tabular}{|c|c|c|c|c|}
\hline Clinical Topics & MOOC & ATT & BTT & + \\
\hline Airway management & + & + & + & + \\
\hline Chest injury & + & + & + & + \\
\hline Fracture stabilization & + & + & + & + \\
\hline Hemorrhage control & + & + & + & + \\
\hline Cervical spine immobilization & + & + & + & + \\
\hline Vital signs and shock & + & + & + & + \\
\hline Scene management & + & + & + & + \\
\hline Triage & + & + & + \\
\hline Extrication & + & + & + \\
\hline IV access & + & + & + \\
\hline Mass casualty & + & + & + \\
\hline UED and cardiac drug administration & & + & + \\
\hline Invasive airway management & & + & + \\
\hline Chest tube insertion & & + & + \\
\hline
\end{tabular}

\section{Application in the Global Context of Trauma Training}

The Global Trauma Initiative Program has successfully established 18 multi-center collaborative sites across India and the USA and trains over 10000 first responders, annually; ranging from local policemen to medical professionals, since its onset in 2008. Furthermore, the virtual component of the BTT phase also assesses trainees with potential to become a trainer of the 'hands on training' component within each respective center. In other words, the model facilitates self-sufficiency and ensures the growth of both trainees and trainers. Next phase of the program is 
use of comics and use of augmented reality and virtual reality for scaling and self-perpetuating expansion. Easy accessibility and the potential to expand the role of non-medical personnel allows such a design to be tailored to fit the needs of multiple global contexts, ranging from isolated underdeveloped areas to robust understaffed urbanized settings. A recent study in China highlights the rapidly growing need for basic trauma training and "targeted training" in urbanized cities across the globe [9]. Various others reiterate the necessity of a standardized acute response to minimize the high rate of mortality occurring within the first few moments after an accident [10-12]. The integration of virtual training programs tailored towards both the setting as well as the background of the trainee has the potential to drastically improve trauma morbidity and mortality rates. Ultimately, the goal of any effort is to lessen the burden of local hospitals and medical professionals as well as minimize expenses due to a delay in providing care. Through the studies conducted by Vyas as well as others in the field, it is becoming increasingly evident that harnessing the exponential growth of technology in the health care sector will help effectively streamline the trauma epidemic.

\section{References}

1. Elmqvist C, Brunt D, Fridlund B, Ekebergh M (2010) Being first on the scene of an accident--experiences of 'doing' prehospital emergency care. Scandinavian journal of caring sciences 24(2): 266-273.

2. Ortiz Figueroa F, Moftakhar Y, Dobbins Iv AL, Khan R, Dasgupta R, et al (2016) Trauma Boot Camp: A Simulation-Based Pilot Study. Cureus 8(1): e463.

\section{ISSN: 2574-1241}

DOI: 10.26717/BJSTR.2019.21.003545

Dinesh Vyas. Biomed J Sci \& Tech Res

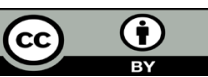

This work is licensed under Creative Commons Attribution 4.0 License

Submission Link: https://biomedres.us/submit-manuscript.php
3. Gillman LM, Widder S, Clement J, Engels PT, Paton Gay JD, et al. (2016) Trauma simulation in bilingual Canada: Insurmountable barrier or unexpected strength? Insights from the first bilingual S.T.A.R.T.T. course. Canadian journal of surgery Journal canadien de chirurgie 59(2): 80-82.

4. Dinesh Vyas MD, Michael Hollis BS, Rohit Abraham MAT, Siddharth Chandra, Ajai Malhotra MD, et al. (2016) Prehospital care training in a rapidly developing economy: a multi-institutional study. Journal of Surgical Research 203(1): 22-27.

5. Fernandez GL, Page DW, Coe NP, Lee PC, Patterson LA, et al. (2012) Boot cAMP: educational outcomes after 4 successive years of preparatory simulation-based training at onset of internship. Journal of surgical education 69(2): 242-248.

6. Rofe G, Lissak A, Brandes Klein O, Segev E, Paz M, et al. (2015) [The Role of Simulation in Surgical Training--a New Era]. Harefuah 154(6): 398403 .

7. Dinesh Vyas, Rohit Abraham, Mayur Narayan, Arpita Vyas (2015) Strategically Leapfrogging Education in Prehospital Trauma Management: Four-Tiered Training Protocols. American Journal of Robotic Surgery 2: 9-15.

8. Aekka A, Abraham R, Hollis M, Boudiab E, Laput G, et al. (2015) Prehospital trauma care education for first responders in India. The Journal of surgical research 197(2): 331-338.

9. Jiang B (2015) The challenges faced in the field of trauma care in China. Zhonghua wai ke za zhi [Chinese journal of surgery] 53(6): 401-404.

10. Callese TE, Richards CT, Shaw P, Schuetz SJ, Issa N, et al. (2014) Layperson trauma training in low- and middle-income countries: a review. The Journal of surgical research 190(1): 104-110.

11. Murad MK, Husum H (2010) Trained lay first responders reduce trauma mortality: a controlled study of rural trauma in Iraq. Prehospital and disaster medicine 25(6): 533-539.

12. Stueven HA, Waite EM, Troiano P, Mateer JR (1989) Prehospital cardiac arrest--a critical analysis of factors affecting survival. Resuscitation 17(3): 251-259.

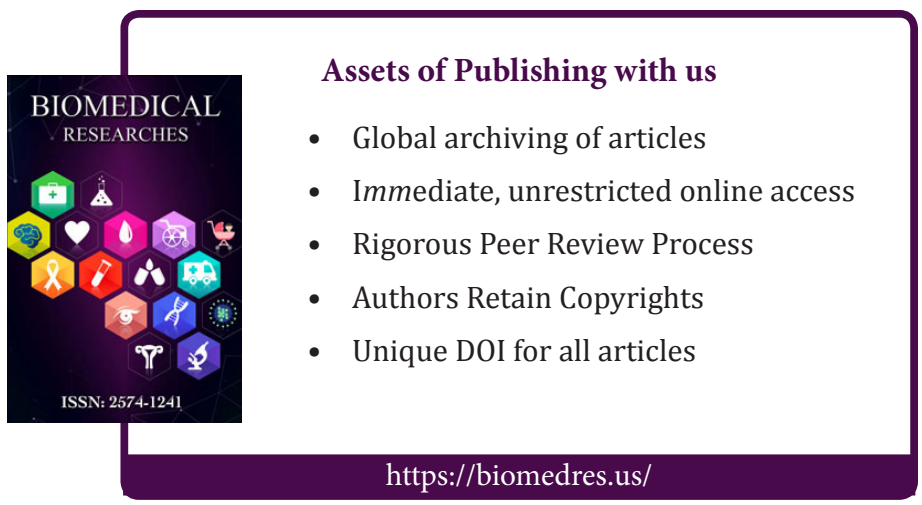

